\title{
ESTIMATES OF BEST APPROXIMATIONS OF FUNCTIONS WITH LOGARITHMIC SMOOTHNESS IN THE LORENTZ SPACE WITH ANISOTROPIC NORM ${ }^{1}$
}

\author{
Gabdolla Akishev \\ L.N. Gumilyov Eurasian National University, \\ 2 Pushkin str., Nur-Sultan, 010008, Kazakhstan \\ Ural Federal University, \\ 19 Mira str., Ekaterinburg, 620002, Russia \\ akishev_g@mail.ru
}

\begin{abstract}
In this paper, we consider the anisotropic Lorentz space $L_{\bar{p}, \bar{\theta}}^{*}\left(\mathbb{I}^{m}\right)$ of periodic functions of $m$ variables. The Besov space $B_{\bar{p}, \bar{\theta}}^{(0, \alpha, \tau)}$ of functions with logarithmic smoothness is defined. The aim of the paper is to find an exact order of the best approximation of functions from the class $B_{\bar{p}, \bar{\theta}}^{(0, \alpha, \tau)}$ by trigonometric polynomials under different relations between the parameters $\bar{p}, \bar{\theta}$, and $\tau$.

The paper consists of an introduction and two sections. In the first section, we establish a sufficient condition for a function $f \in L_{\bar{p}, \bar{\theta}^{(1)}}^{*}\left(\mathbb{I}^{m}\right)$ to belong to the space $L_{\bar{p}, \theta^{(2)}}^{*}\left(\mathbb{I}^{m}\right)$ in the case $1<\theta^{2}<\theta_{j}^{(1)}, j=1, \ldots, m$, in terms of the best approximation and prove its unimprovability on the class $E_{\bar{p}, \bar{\theta}}^{\lambda}=\left\{f \in L_{\bar{p}, \bar{\theta}}^{*}\left(\mathbb{I}^{m}\right): E_{n}(f)_{\bar{p}, \bar{\theta}} \leq \lambda_{n}\right.$, $n=0,1, \ldots\}$, where $E_{n}(f)_{\bar{p}, \bar{\theta}}$ is the best approximation of the function $f \in L_{\bar{p}, \bar{\theta}}^{*}\left(\mathbb{I}^{m}\right)$ by trigonometric polynomials of order $n$ in each variable $x_{j}, j=1, \ldots, m$, and $\lambda=\left\{\lambda_{n}\right\}$ is a sequence of positive numbers $\lambda_{n} \downarrow 0$ as $n \rightarrow+\infty$. In the second section, we establish order-exact estimates for the best approximation of functions from the class $B_{\bar{p}, \bar{\theta}^{(1)}}^{(0, \alpha, \tau)}$ in the space $L_{\bar{p}, \theta^{(2)}}^{*}\left(\mathbb{I}^{m}\right)$.
\end{abstract}

Key words: Lorentz space, Nikol'skii-Besov class, Best approximation.

\section{Introduction}

Let $\bar{x}=\left(x_{1}, \ldots, x_{m}\right) \in \mathbb{R}^{m}, \mathbb{I}^{m}=[0,2 \pi]^{m}, \bar{p}=\left(p_{1}, \ldots, p_{m}\right)$, and $\bar{\theta}=\left(\theta_{1}, \ldots, \theta_{m}\right)$, where $p_{j} \in(1, \infty)$ and $\theta_{j} \in[1, \infty)$ for $j=1,2, \ldots, m$. Denote by $L_{\bar{p}, \bar{\theta}}^{*}\left(\mathbb{I}^{m}\right)$ the Lorentz space of real-valued functions $f(\bar{x})$ that are $2 \pi$-periodic in each variable and

$$
\|f\|_{\bar{p}, \bar{\theta}}^{*}=\left\{\int_{0}^{2 \pi} t_{m}^{\frac{\theta_{m}}{p_{m}}-1}\left[\ldots\left[\int_{0}^{2 \pi}\left(f^{*_{1}, \ldots, *_{m}}\left(t_{1}, \ldots, t_{m}\right)\right)^{\theta_{1}} t_{1}^{\frac{\theta_{1}}{p_{1}}-1} d t_{1}\right]^{\frac{\theta_{2}}{\theta_{1}}} \ldots\right]^{\frac{\theta_{m}}{\theta_{m}-1}} d t_{m}\right\}^{1 / \theta_{m}}<+\infty,
$$

where $f^{*_{1}, \ldots, *_{m}}$ is a nonincreasing rearrangement of the function $\left|f\left(x_{1}, \ldots, x_{m}\right)\right|$ in each of the variables $x_{j}$ whereas the other variables are fixed (see $[8,18]$ ).

In the case $p_{1}=\cdots=p_{m}=\theta_{1}=\cdots=\theta_{m}=p$, the Lorentz space $L_{\bar{p}, \bar{\theta}}^{*}\left(\mathbb{I}^{m}\right)$ coincides with the Lebesgue space $L_{p}\left(\mathbb{I}^{m}\right)$ with the norm

$$
\|f\|_{p}=\left[\int_{0}^{2 \pi} \ldots \int_{0}^{2 \pi}\left|f\left(x_{1}, \ldots, x_{m}\right)\right|^{p} d x_{1} \ldots d x_{m}\right]^{1 / p}
$$

\footnotetext{
${ }^{1}$ This work was supported by the Competitiveness Enhancement Program of the Ural Federal University (Enactment of the Government of the Russian Federation of March 16, 2013 no. 211, agreement no. 02.A03. 21.0006 of August 27, 2013).
} 
where $p \in[1,+\infty)$.

Instead of $L_{\bar{p}, \bar{\theta}}^{*}\left(\mathbb{I}^{m}\right)$, we will write $L_{p, \theta}^{*}\left(\mathbb{I}^{m}\right)$ in the case $p_{1}=\cdots=p_{m}=p$ and $\theta_{1}=\cdots=\theta_{m}=\theta$ and $L_{\bar{p}, \theta^{(2)}}^{*}\left(\mathbb{I}^{m}\right)$ if $\bar{p}=\left(p_{1}, \ldots, p_{m}\right)$ and $\theta_{1}=\cdots=\theta_{m}=\theta^{(2)}$.

Given a natural number $M$, consider the set

$$
\square_{M}=\left\{\bar{k}=\left(k_{1}, \ldots, k_{m}\right) \in \mathbb{Z}^{m}:\left|k_{j}\right|<M, j=1, \ldots, m\right\} .
$$

Consider the multiple Dirichlet kernel

$$
D_{\square_{M}}(\bar{x})=\sum_{\bar{k} \in \square_{M}} e^{i\langle\bar{k}, \bar{x}\rangle}, \quad \bar{x} \in \mathbb{I}^{m},
$$

and its convolution with a function $f \in L_{\bar{p}, \bar{\theta}}^{*}\left(\mathbb{I}^{m}\right)$ :

$$
\sigma_{s}(f, \bar{x})=\int_{\mathbb{I}^{m}} f(\bar{y})\left(D_{\square_{2^{s}}}(\bar{x}-\bar{y})-D_{\square_{2^{s-1}}}(\bar{x}-\bar{y})\right) d \bar{y},
$$

where $s \in \mathbb{N}_{0}=\mathbb{N} \cup\{0\}$ and $\mathbb{N}$ is the set of positive integers.

Let $M \in \mathbb{N}_{0}$, and let $T_{M}(\bar{x})=\sum_{\bar{k} \in \square_{M}} a_{\bar{k}} e^{i\langle\bar{k}, \bar{x}\rangle}$ be a trigonometric polynomial of order $M$ in each variable $x_{j}, j=1, \ldots, m$. Denote by $\mathfrak{F}_{\square_{M}}$ the set of all such polynomials.

Let $E_{M, \ldots, M}(f)_{\bar{p}, \bar{\theta}}=\inf _{T \in \mathfrak{F}_{\square_{M}}}\|f-T\|_{\bar{p}, \bar{\theta}}^{*}$ be the best approximation of a function $f \in L_{\bar{p}, \bar{\theta}}^{*}\left(\mathbb{I}^{m}\right)$ by the set $\mathfrak{F}_{\square_{M}}$. Sometimes, we will use the notation $E_{M}(f)_{\bar{p}, \bar{\theta}}$ instead of $E_{M, \ldots, M}(f)_{\bar{p}, \bar{\theta}}$. For a given class $F \subset L_{\bar{p}, \bar{\theta}}^{*}\left(\mathbb{I}^{m}\right)$, let $E_{M}(F)_{\bar{p}, \bar{\theta}}=\sup _{f \in F} E_{M}(f)_{\bar{p}, \bar{\theta}}$.

Let $\alpha \geq 0, \gamma \in(-\infty,+\infty)$, and $0<\tau<\infty$. Denote by $\mathbb{A}_{\bar{p}, \bar{\theta}}^{(\alpha, \gamma, \tau)}$ the space of all functions $f \in L_{\bar{p}, \bar{\theta}}^{*}\left(\mathbb{I}^{m}\right)$ such that the quasi-norm (see $[9,20]$ )

$$
\|f\|_{\mathbb{A}_{\bar{p}, \bar{\theta}}^{(\alpha, \gamma, \tau)}}=\left[\sum_{n=1}^{\infty} n^{-1}\left(n^{\alpha}(1+\log n)^{\gamma} E_{n}(f)_{\bar{p}, \bar{\theta}}\right)^{\tau}\right]^{1 / \tau}
$$

is finite, where $\log a$ is the logarithm of the number $a$ to the base 2 .

If $\tau=\infty$, then

$$
\|f\|_{\mathbb{A}_{\bar{p}, \bar{\theta}}^{\alpha, \gamma}, \tau}=\sup _{n \geq 1} n^{\alpha}(1+\log n)^{\gamma} E_{n}(f)_{\bar{p}, \bar{\theta}}<\infty .
$$

It is known that $\mathbb{A}_{\bar{p}, \bar{\theta}}^{(\alpha, \gamma) \tau)}$ is a quasi-Banach space (see $\left.[9,10,20]\right)$. It is called an approximate space (see [11]).

In the anisotropic Lorentz space, we consider the space $B_{\bar{p}, \bar{\theta}}^{(0, \alpha, \tau)}, 1 \leq \tau \leq \infty$, of all functions $f \in L_{\bar{p}, \bar{\theta}}^{*}\left(\mathbb{I}^{m}\right)$ representable in the form of series

$$
\sum_{n=0}^{\infty} Q_{2^{2^{n}}}(f, \bar{x}), \quad Q_{2^{2^{n}}}(f) \in \mathfrak{F}_{\square^{2^{n}}}
$$

and such that

$$
\left[\sum_{n=0}^{\infty}\left(2^{n \alpha}\left\|Q_{2^{2^{n}}}(f)\right\|_{\bar{p}, \bar{\theta}}^{*}\right)^{\tau}\right]^{1 / \tau}<+\infty
$$

for $1 \leq \tau<\infty$ and

$$
\sup _{n \in \mathbb{N}_{0}} 2^{n \alpha}\left\|Q_{2^{2^{n}}}(f)\right\|_{\bar{p}, \bar{\theta}}^{*}<\infty
$$


for $\tau=\infty$. The infimum of expression (1.2) over all representations (1.1) defines a quasi-norm in this space:

$$
\|f\|_{B_{\bar{p}, \bar{\theta}}^{(0, \alpha, \tau)}}=\inf \left[\sum_{n=0}^{\infty}\left(2^{n \alpha}\left\|Q_{2^{2^{n}}}(f)\right\|_{\bar{p}, \bar{\theta}}^{*}\right)^{\tau}\right]^{1 / \tau}
$$

The space $B_{\bar{p}, \bar{\theta}}^{(0, \alpha)}$ is called the Besov space with logarithmic smoothness. In $B_{\bar{p}, \bar{\theta}}^{(0, \alpha, \tau)}$, we consider the unit ball

$$
\mathbb{B}_{\bar{p}, \bar{\theta}}^{(0, \alpha, \tau)}=\left\{f \in L_{\bar{p}, \bar{\theta}}^{*}\left(\mathbb{I}^{m}\right):\|f\|_{B_{\bar{p}, \bar{\theta}}^{(0, \alpha, \tau)}} \leq 1\right\}
$$

It is known that $f \in \mathbb{B}_{\bar{p}, \bar{\theta}}^{(0, \gamma+1 / \tau, \tau)}$ if and only if $f \in \mathbb{A}_{\bar{p}, \bar{\theta}}^{(0, \gamma, \tau)}$ (see [10]).

The main aim of the present paper is to obtain an exact order of the best approximation of the function classes $\mathbb{A}_{\bar{p}, \bar{\theta}^{(1)}}^{(0, \gamma, \tau)}$ and $\mathbb{B}_{\bar{p}, \bar{\theta}^{(1)}}^{(0, \gamma, \tau)}$ in anisotropic Lorentz spaces.

In the one-dimensional case, sufficient conditions for a function $f \in L_{p}\left(I^{1}\right)$ to belong to the space $L_{q}\left(\mathbb{I}^{1}\right)$ for $1 \leq p<q<\infty$ in terms of the best approximation and the modulus of continuity were established by P.L. Ul'ynov [30]. This study was continued by V.I. Kolyada [15], V.A. Andrienko [5], N. Temirgaliev [27, 28], E.A. Storozhenko [26], M.F. Timan, P. Oswald, L. Leindler, S.V. Lapin, B.V. Simonov, and others (see the references in [16]).

N. Temirgaliev established [28] a necessary and sufficient condition for a univariate function $f \in L_{p}\left(\mathbb{I}^{1}\right)$ to belong to the Lorentz space $L_{q, \theta}\left(\mathbb{I}^{1}\right)$ in terms of the modulus of continuity for $1 \leq \theta<p<\infty$. L.A. Sherstneva studied [22] this problem in terms of the best approximation of a function. Such problems in the Lorentz space were investigated in [1, 4, 23].

Problems of estimating various approximative characteristics of function classes are well known and a survey of the results on this topic is given in $[12,29]$. In particular, in the Lebesgue space $L_{p}\left(\mathbb{I}^{m}\right)$, exact estimates of the best approximation of functions of the Besov class $B_{p, \bar{\theta}^{(1)}}^{r}$ were established by A.S. Romanyuk [21]. In the case $\theta_{j}^{(1)}=p_{j}=p, j=1, \ldots, m$, estimates of approximative characteristics of the class $\mathbb{B}_{\bar{p}, \bar{\theta}^{(1)}}^{0, \alpha}$ were obtained by S.A. Stasyuk [24, 25]. In [13], the embedding and characterization problems of the Besov space with logarithmic smoothness in the Lebesgue space $L_{p}\left(\mathbb{I}^{m}\right)$ were investigated.

Exact estimates of best approximations of functions from the Besov class in the Lorentz space with a mixed norm were obtained in $[2,6,7]$.

The present paper consists of the introduction and two sections. In Section 1, we establish a sufficient condition for a function $f \in L_{\bar{p}, \bar{\theta}^{*}}^{*}\left(\mathbb{I}^{m}\right)$ to belong to the space $L_{\bar{p}, \theta^{(2)}}^{*}\left(\mathbb{I}^{m}\right), \theta^{(2)}<\theta_{j}^{(1)}$, $j=1, \ldots, m$, and prove its accuracy on the class

$$
E_{\bar{p}, \bar{\theta}}^{\lambda}=\left\{f \in L_{\bar{p}, \bar{\theta}}^{*}\left(\mathbb{I}^{m}\right): E_{n}(f)_{\bar{p}, \bar{\theta}} \leq \lambda_{n}, n=0,1, \ldots\right\}
$$

where $\lambda=\left\{\lambda_{n}\right\}$ is a sequence of positive numbers $\lambda_{n} \downarrow 0$ as $n \rightarrow+\infty$.

In the case $p_{j}=\theta_{j}=p, j=1, \ldots, m$, V.I. Kolyada proved [15] a necessary and sufficient condition for the embedding of classes $E_{p}^{\lambda}$ in the space $L_{q}\left(\mathbb{I}^{1}\right), 1 \leq p<q$.

In Section 2, we establish order-exact estimates of the value $E_{n}\left(\mathbb{B}_{\bar{p}, \bar{\theta}^{(1)}}^{(0, \gamma)}\right)_{\bar{q}, \bar{\theta}^{(2)}}$ under various relations between coordinates of the parameters $\bar{p}, \bar{\theta}^{(1)}, \bar{q}, \bar{\theta}^{(2)}, \tau$ (see Theorems 5 and 6 ).

The notation $A(y) \asymp B(y)$ means that there exists positive constants $C_{1}$ and $C_{2}$ such that $C_{1} A(y) \leq B(y) \leq C_{2} A(y)$. If $B(y) \leq C_{2} A(y)$ or $A(y) \geq C_{1} B(y)$, then we write $B(y)<<A(y)$ and $A(y)>>B(y)$, respectively. 


\section{Conditions for embedding classes in the Lorentz space}

Theorem 1 [19, Theorem 10]. Let $1 \leq p_{j}<+\infty$ and $1 \leq \theta_{j}<q_{j}<+\infty$ for $j=1, \ldots, m$, let $\bar{p}=\left(p_{1}, \ldots, p_{m}\right)$ and $\bar{q}=\left(q_{1}, \ldots, q_{m}\right)$, and let $\bar{\theta}=\left(\theta_{1}, \ldots, \theta_{m}\right)$. Then a trigonometric polynomial

$$
T_{\bar{n}}(\bar{x})=\sum_{k_{1}=-n_{1}}^{n_{1}} \cdots \sum_{k_{m}=-n_{m}}^{n_{m}} b_{\bar{k}} e^{i\langle\bar{x}, \bar{k}\rangle}
$$

satsfies the following inequality:

$$
\left\|T_{\bar{n}}\right\|_{\bar{p}, \bar{\theta}}^{*} \leq C(p, q, \theta) \prod_{j=1}^{m}\left(\ln \left(1+n_{j}\right)\right)^{1 / \theta_{j}-1 / q_{j}}\left\|T_{\bar{n}}\right\|_{\bar{p}, \bar{q}}^{*} .
$$

Lemma 1. Let $1<p_{j}<\infty$ and $1<q_{2}<q_{j}^{(1)}<+\infty$ for $j=1, \ldots, m$. Let $\left\{u_{n}\right\}$ be a sequence of non-negative measurable functions on the cube $\mathbb{I}^{m}=[0,2 \pi]^{m}$ such that

(1)

$$
\left\|u_{n}\right\|_{\bar{p}, \bar{q}^{(1)}}^{*} \leq \varepsilon_{n}, \quad \varepsilon_{n+1} \leq \beta \varepsilon_{n}, \quad \beta \in(0,1) ;
$$

(2) there exists a sequence of positive numbers $\left\{\Delta_{n}\right\}$ such that

$$
\left\|u_{n}\right\|_{p, \theta}^{*} \leq C \Delta_{n}^{\sum_{j=1}^{m}\left(1 / \theta_{j}-1 / q_{j}^{(1)}\right)} \varepsilon_{n}, \quad n=1,2,3, \ldots
$$

for any $\theta_{j} \in\left(0, q_{j}^{(1)}\right), j=1, \ldots, m$.

Then the inequality

$$
\|f\|_{p, q_{2}}^{*} \leq C\left\{\sum_{n=1}^{\infty} \Delta_{n}^{\sum_{j=1}^{m}\left(1 / q_{2}-1 / q_{j}^{(1)}\right)} \varepsilon_{n}^{q_{2}}\right\}^{1 / q_{2}}
$$

holds for every function of the form $f(\bar{x})=\sum_{n=1}^{\infty} u_{n}(\bar{x})$.

This lemma is proved by V.I. Kolyada's method (see [15, Proof of Lemma 4]) as in [3].

Remark 1. Lemma 1 was proved by L.A. Sherstneva [22, Lemma 13] in the one-dimensional case and by the author [3] in the multi-dimensional case for $q_{1}^{(1)}=\cdots=q_{m}^{(1)}$.

Now, let us consider a condition for a function $f \in L_{\bar{p}, \bar{\theta}(1)}^{*}\left(\mathbb{I}^{m}\right)$ to belong to the space $L_{\bar{p}, \theta^{(2)}}^{*}\left(\mathbb{I}^{m}\right)$, $1<\theta^{(2)}<\theta_{j}^{(1)}<+\infty, j=1, \ldots, m$.

Theorem 2. Let $1<\theta^{(2)}<\theta_{j}^{(1)}<+\infty$ and $1<p_{j}<\infty$ for $j=1, \ldots$, m, and let $\bar{\theta}^{(1)}=$ $\left(\theta_{1}^{(1)}, \ldots, \theta_{m}^{(1)}\right)$. Assume that $f \in L_{\bar{p}, \bar{\theta}^{(1)}}^{*}\left(\mathbb{I}^{m}\right)$ and

$$
\sum_{n=2}^{\infty} \frac{(\ln n)^{\theta^{(2)} \sum_{j=1}^{m}\left(1 / \theta^{(2)}-1 / \theta_{j}^{(1)}\right)-1}}{n} E_{n, \ldots, n}^{\theta^{(2)}}(f)_{\bar{p}, \bar{\theta}^{(1)}}<+\infty .
$$

Then $f \in L_{\bar{p}, \theta^{(2)}}^{*}\left(\mathbb{I}^{m}\right)$ and

$$
\|f\|_{\bar{p}, \theta^{(2)}}^{*}<<\left\{\|f\|_{\bar{p}, \theta^{(1)}}^{*}+\left[\sum_{k=2}^{\infty} \frac{(\ln (k+1))^{\theta^{(2)}} \sum_{j=1}^{m}\left(1 / \theta^{(2)}-1 / \theta_{j}^{(1)}\right)-1}{k} E_{k, \ldots, k}^{\theta^{(2)}}(f)_{\bar{p}, \bar{\theta}^{(1)}}\right]^{1 / \theta^{(2)}}\right\},
$$




$$
\begin{aligned}
& E_{n, \ldots, n}(f)_{\bar{p}, \theta^{(2)}}<<\left\{(\ln (n+1))^{\sum_{j=1}^{m}\left(1 / \theta^{(2)}-1 / \theta_{j}^{(1)}\right)} E_{n, \ldots, n}(f)_{\bar{p}, \bar{\theta}^{(1)}}+\right. \\
& \left.+\left[\sum_{k=n+1}^{\infty} \frac{(\ln (k+1))^{\theta^{(2)} \sum_{j=1}^{m}\left(1 / \theta^{(2)}-1 / \theta_{j}^{(1)}\right)-1}}{k} E_{k, \ldots, k}^{\theta^{(2)}}(f)_{\bar{p}, \bar{\theta}^{(1)}}\right]^{1 / \theta^{(2)}}\right\} .
\end{aligned}
$$

P r o o f. Since $E_{n, \ldots, n}(f)_{\bar{p}, \bar{\theta}^{(1)}} \equiv \varepsilon_{n} \downarrow 0$ as $n \rightarrow+\infty$ for every function $f \in L_{\bar{p}, \bar{\theta}^{(1)}}^{*}\left(\mathbb{I}^{m}\right)$, $1<p_{j}, \theta_{j}^{(1)}<+\infty, j=1, \ldots, m$, there exists a numerical sequence $\left\{n_{\nu}\right\}$ such that (see [15, Sect. 2])

$$
\varepsilon_{n_{\nu+1}}<\frac{1}{2} \varepsilon_{n_{\nu}}, \quad \varepsilon_{n_{\nu+1}-1} \geq \frac{1}{2} \varepsilon_{n_{\nu}}, \quad \nu=1,2, \ldots .
$$

Let $T_{n}(f, \bar{x})$ be a trigonometric polynomial of the best approximation of a function $f \in L_{\bar{p}, \bar{\theta}^{(1)}}^{*}\left(\mathbb{I}^{m}\right), \quad 1<p_{j}, \theta_{j}^{(1)}<+\infty, j=1, \ldots, m$. Consider the series

$$
T_{n_{1}}(f, \bar{x})+\sum_{\nu=1}^{\infty}\left(T_{n_{\nu+1}}(f, \bar{x})-T_{n_{\nu}}(f, \bar{x})\right) .
$$

Let us prove the convergence of this series in the norm of the space $L_{\bar{p}, \theta^{(2)}}^{*}\left(\mathbb{I}^{m}\right)$. Suppose that

$$
u_{\nu}(\bar{x})=\left|T_{n_{\nu+1}}(f, \bar{x})-T_{n_{\nu}}(f, \bar{x})\right|, \quad \nu=0,1, \ldots
$$

Then

$$
\left\|u_{\nu}\right\|_{\bar{p}, \bar{\theta}^{(1)}}^{*} \leq 2 \varepsilon_{\nu}, \quad \nu=0,1, \ldots
$$

and, by Theorem 1 ,

$$
\left\|u_{\nu}\right\|_{\bar{p}, \bar{\tau}}^{*}<<\left(\ln n_{\nu+1}\right)^{\sum_{j=1}^{m}\left(1 / \tau_{j}-1 / \theta_{j}^{(1)}\right)} \varepsilon_{\nu}
$$

for any $\tau_{j} \in\left(0, \theta_{j}^{(1)}\right), j=1, \ldots, m$. Hence, by Lemma 1 , we obtain

$$
\begin{aligned}
& \left\|\sum_{\nu=k+1}^{l}\left(T_{n_{\nu+1}}(f)-T_{n_{\nu}}(f)\right)\right\|_{\bar{p}, \theta^{(2)}}^{*} \leq\left\|\sum_{\nu=k+1}^{l} u_{\nu}\right\|_{\bar{p}, \theta^{(2)}}^{*}<< \\
& <<\left\{\sum_{\nu=k+1}^{l}\left(\ln n_{\nu+1}\right)^{\theta^{(2)} \sum_{j=1}^{m}\left(1 / \theta^{(2)}-1 / \theta_{j}^{(1)}\right)} \varepsilon_{\nu}^{\theta^{(2)}}\right\}^{1 / \theta^{(2)}} .
\end{aligned}
$$

Condition (2.1) implies that

$$
\sum_{\nu=1}^{\infty}\left(\ln n_{\nu+1}\right)^{\theta^{(2)} \sum_{j=1}^{m}\left(1 / \theta^{(2)}-1 / \theta_{j}^{(1)}\right)} \varepsilon_{n_{\nu}}^{\theta^{(2)}}<+\infty .
$$

It follows from (2.5) and (2.6) that series (2.4) converges to a function $g \in L_{\bar{p}, \theta^{(2)}}^{*}\left(\mathbb{I}^{m}\right)$ in the norm. It is easy to see that $g(\bar{x})=f(\bar{x})$ almost everywhere on $\mathbb{I}^{m}$. Hence, $f \in L_{\bar{p}, \theta^{(2)}}^{*}\left(\mathbb{I}^{m}\right)$. Setting $k=0$ in (2.5), we get

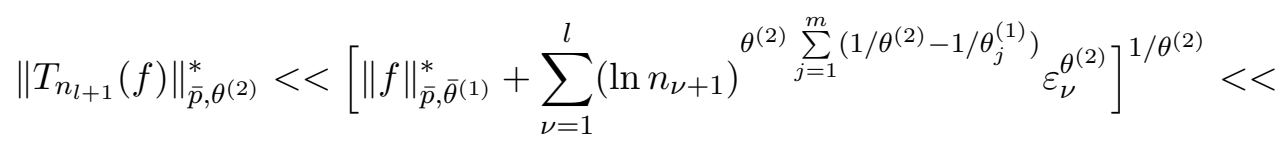




$$
<<\left\{\|f\|_{\bar{p}, \bar{\theta}^{(1)}}^{*}+\left[\sum_{n=2}^{\infty} \frac{(\ln (n+1))^{\theta^{(2)}} \sum_{j=1}^{m}\left(1 / \theta^{(2)}-1 / \theta_{j}^{(1)}\right)-1}{n} E_{n, \ldots, n}^{\theta^{(2)}}(f)_{\bar{p}, \bar{\theta}^{(1)}}\right]^{1 / \theta^{(2)}}\right\} .
$$

By tending $l$ to $+\infty$ in this inequality, we obtain

$$
\|f\|_{\bar{p}, \theta^{(2)}}^{*}<<\left\{\|f\|_{\bar{p}_{,} \bar{\theta}^{(1)}}^{*}+\left[\sum_{n=2}^{\infty} \frac{(\ln (n+1))^{\theta^{(2)}} \sum_{j=1}^{m}\left(1 / \theta^{(2)}-1 / \theta_{j}^{(1)}\right)-1}{n} E_{n, \ldots, n}^{\theta^{(2)}}(f)_{\bar{p}_{,} \bar{\theta}^{(1)}}\right]^{1 / \theta^{(2)}}\right\} .
$$

Thus, inequality (2.2) is proved.

Applying inequality (2.2) to the function $f-T_{n}(f) \in L_{\bar{p}, \theta^{(2)}}^{*}\left(\mathbb{I}^{m}\right)$, it is easy to prove inequality (2.3). The proof of Theorem 2 is complete.

Let us prove that condition $(2.1)$ is exact on the classes $E_{\bar{p}, \bar{\theta}^{(1)}}^{\lambda}$.

Theorem 3. Let $1<p_{j}<\infty$ and $1<\theta^{(2)}<\theta_{j}^{(1)}<+\infty$ for $j=1, \ldots, m$. The following condition is necessary and sufficient for the inclusion $E_{\bar{p}, \bar{\theta}(1)}^{\lambda} \subset L_{\bar{p}, \theta^{(2)}}^{*}\left(\mathbb{I}^{m}\right)$ :

$$
\sum_{n=2}^{\infty} \frac{(\ln n)^{\theta^{(2)}} \sum_{j=1}^{m}\left(1 / \theta^{(2)}-1 / \theta_{j}^{(1)}\right)-1}{n} \lambda_{n}^{\theta^{(2)}}<+\infty .
$$

P r o o f. The sufficiency of condition (2.7) follows from Theorem 2. Let us prove the necessity. Let $E_{\bar{p}, \bar{\theta}^{(1)}}^{\lambda} \subset L_{\bar{p}, \theta^{(2)}}^{*}\left(\mathbb{I}^{m}\right)$. Assume that condition (2.7) is violated, i.e.,

$$
\sum_{n=2}^{\infty} \frac{(\ln n)^{\theta^{(2)}} \sum_{j=1}^{m}\left(1 / \theta^{(2)}-1 / \theta_{j}^{(1)}\right)-1}{n} \lambda_{n}^{\theta^{(2)}}=+\infty
$$

We choose a sequence of numbers $\left\{\nu_{k}\right\}$ with the following properties (see [15]):

$$
\lambda_{\nu_{k+1}}<\frac{1}{2} \lambda_{\nu_{k}}, \quad \lambda_{\nu_{k+1}-1} \geq \frac{1}{2} \lambda_{\nu_{k}} .
$$

Since the function $(\ln x)^{\beta} / x$ with $\beta \in \mathbb{R}$ decreases to 0 as $x \rightarrow+\infty$, we have

$$
\begin{gathered}
\sum_{n=\nu_{k}+1}^{\nu_{k+1}} \frac{(\ln n)^{\theta^{(2)} \sum_{j=1}^{m}\left(1 / \theta^{(2)}-1 / \theta_{j}^{(1)}\right)-1}}{n} \leq \sum_{n=\nu_{k}+1}^{\nu_{k+1}} \frac{\left(\ln \left(n-\nu_{k}+1\right)\right)^{\theta^{(2)} \sum_{j=1}^{m}\left(1 / \theta^{(2)}-1 / \theta_{j}^{(1)}\right)-1}}{n-\nu_{k}}<< \\
<<\left(\ln \left(\nu_{k+1}-\nu_{k}+1\right)\right)^{\theta^{(2)} \sum_{j=1}^{m}\left(1 / \theta^{(2)}-1 / \theta_{j}^{(1)}\right)} .
\end{gathered}
$$

Thus, (2.8) implies that

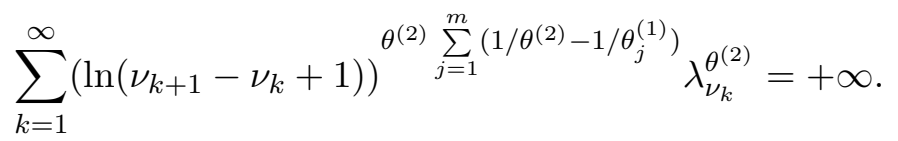

Let us consider the function

$$
f_{0}(\bar{x})=\sum_{k=0}^{\infty} \lambda_{\nu_{k}}\left(\ln \left(\nu_{k+1}-\nu_{k}+1\right)\right)^{-\sum_{j=1}^{m} 1 / \theta_{j}^{(1)}} \tau_{k}(\bar{x}),
$$


where

$$
\tau_{k}(\bar{x})=\prod_{j=1}^{m} \sum_{n_{j}=\nu_{k}+1}^{\nu_{k+1}}\left(n_{j}-\nu_{k}\right)^{\frac{1}{p_{j}}-1} \sin n_{j} x_{j} .
$$

It is known that (see [22])

$$
\left\|\tau_{k}\right\|_{\bar{p}^{\prime}, \bar{\theta}^{(1)}} \asymp\left(\ln \left(\nu_{k+1}-\nu_{k}+1\right)\right)^{\sum_{j=1}^{m} 1 / \theta_{j}^{(1)}}, \quad 1<p_{j}, \theta_{j}^{(1)}<+\infty, \quad j=1, \ldots, m .
$$

Using this relation and (2.9), we can verify that

$$
\left\|f_{0}\right\|_{\bar{p}, \bar{\theta}^{(1)}}^{*} \leq \sum_{k=0}^{\infty} \lambda_{\nu_{k}}\left(\ln \left(\nu_{k+1}-\nu_{k}+1\right)\right)^{-\sum_{j=1}^{m} 1 / \theta_{j}^{(1)}}\left\|\tau_{k}\right\|_{\bar{p}, \bar{\theta}^{(1)}}^{*} \leq C \sum_{k=0}^{\infty} \lambda_{\nu_{k}}<\infty .
$$

Hence, $f_{0} \in L_{\bar{p}, \bar{\theta}^{(1)}}^{*}\left(\mathbb{I}^{m}\right), 1<p_{j}, \theta_{j}^{(1)}<\infty, j=1, \ldots, m$.

Let a positive integer $n$ satisfy the inequalities $\nu_{l} \leq n<\nu_{l+1}$. Then, by the best approximation property and according to relation (2.11) and inequality (2.9), we have

$$
\begin{gathered}
E_{n}\left(f_{0}\right)_{\bar{p}, \bar{\theta}^{(1)}} \leq E_{\nu_{l}}\left(f_{0}\right)_{\bar{p}, \bar{\theta}^{(1)}} \leq \sum_{k=l}^{\infty} \lambda_{\nu_{k}}\left(\ln \left(\nu_{k+1}-\nu_{k}+1\right)\right)^{-\sum_{j=1}^{m} 1 / \theta_{j}^{(1)}}\left\|\tau_{k}\right\|_{\bar{p}, \bar{\theta}^{(1)}}^{*}<< \\
<<\sum_{k=l}^{\infty} \lambda_{\nu_{k}}<<\lambda_{\nu_{l}}<<2 \lambda_{\nu_{l+1}-1} \leq C_{0} \lambda_{n} .
\end{gathered}
$$

Hence, $f_{1}=C_{0}^{-1} f_{0} \in E_{\bar{p}, \bar{\theta}^{(1)}}^{\lambda}$.

Let us show that $f_{1} \notin L_{\bar{p}, \theta^{(2)}}^{*}\left(\mathbb{I}^{m}\right), 1<\theta^{(2)}<\infty$. To this end, we consider the function

$$
g_{0}(\bar{x})=\sum_{k=0}^{\infty}\left(\ln \left(\nu_{k+1}-\nu_{k}+1\right)\right)^{\sum_{j=1}^{m} \frac{1-\theta^{(2)}}{\theta_{j}^{(1)}}} \lambda_{\nu_{k}}^{\theta^{(2)}}-1 \xi_{k}(\bar{x}),
$$

where

$$
\xi_{k}(\bar{x})=\prod_{j=1}^{s} \sum_{n_{j}=\nu_{k}+1}^{\nu_{k+1}}\left(n_{j}-\nu_{k}\right)^{\frac{1}{p_{j}^{\prime}}-1} \sin n_{j} x_{j}, \quad p_{j}^{\prime}=\frac{p_{j}}{p_{j}-1}, \quad j=1, \ldots, m .
$$

It is clear that (see (2.11))

$$
\left\|\xi_{k}\right\|_{\bar{p}^{\prime}, \bar{\theta}}^{*} \asymp\left(\ln \left(\nu_{k+1}-\nu_{k}+1\right)\right)^{\sum_{j=1}^{m} 1 / \theta_{j}}, \quad 1<p_{j}<+\infty, \quad 1<\theta_{j}<\infty, \quad j=1, \ldots, m .
$$

Further, in view of the orthogonality of the trigonometric system, for any number $N$, we have

$$
\begin{aligned}
& B_{N} \equiv \int_{\mathbb{I}^{m}} f_{1}(\bar{x}) \sum_{k=0}^{N} \lambda_{\nu_{k}}^{\theta^{(2)}-1}\left(\ln \left(\nu_{k+1}-\nu_{k}+1\right)\right)^{\sum_{j=1}^{m} \frac{1-\theta^{(2)}}{\theta_{j}^{(1)}}} \xi_{k}(\bar{x}) d \bar{x}= \\
& =C \sum_{k=0}^{N}\left[\ln \left(\nu_{k+1}-\nu_{k}+1\right)\right]^{-\theta^{(2)} \sum_{j=1}^{m} 1 / \theta_{j}^{(1)}} \lambda_{\nu_{k}}^{\theta^{(2)}} \prod_{j=1}^{m} \sum_{n_{j}=\nu_{k}+1}^{\nu_{k+1}} \frac{1}{n_{j}-\nu_{k}}>>
\end{aligned}
$$

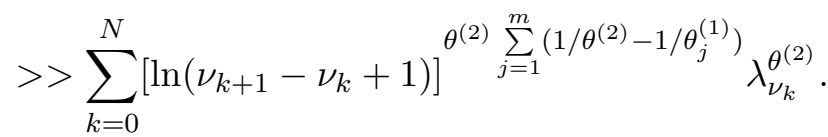


Using the integral Hölder inequality, we obtain

$$
B_{N}<<\left\|f_{1}\right\|_{\bar{p}, \theta^{(2)}}^{*}\left\|\sum_{k=0}^{N}\left(\ln \left(\nu_{k+1}-\nu_{k}+1\right)\right)^{\sum_{j=1}^{m} \frac{1-\theta^{(2)}}{\theta_{j}^{(1)}}} \lambda_{\nu_{k}}^{\theta^{(2)}-1} \xi_{k}\right\|_{\bar{p}^{\prime}, \theta^{(2)^{\prime}}}^{*}
$$

where

$$
\theta^{(2)^{\prime}}=\frac{\theta^{(2)}}{\theta^{(2)}-1}
$$

We set $u_{k}(\bar{x})=\left(\ln \left(\nu_{k+1}-\nu_{k}+1\right)\right)^{\sum_{j=1}^{m} \frac{1-\theta^{(2)}}{\theta_{j}^{(1)}}} \lambda_{\nu_{k}}^{\theta^{(2)}-1}\left|\xi_{k}(\bar{x})\right|$. Then $($ see $(2.11))$

$$
\begin{gathered}
\left\|u_{k}\right\|_{\bar{p}^{\prime}, \frac{\bar{\theta}}{\theta^{(2)}-1}}^{*}<<\lambda_{\nu_{k}}^{\theta^{(2)}-1} \equiv \beta_{k}, \\
\left\|u_{k}\right\|_{\bar{p}^{\prime}, \bar{\tau}}^{*}<<\left[\ln \left(\nu_{k+1}-\nu_{k}+1\right)\right]^{\sum_{j=1}^{m}\left(\frac{1}{\tau_{j}}-\frac{\theta^{(2)}-1}{\theta_{j}^{(1)}}\right)} \beta_{k}, \quad k=0,1, \ldots .
\end{gathered}
$$

Thus, all the conditions of Lemma 1 hold for the sequence of functions $\left\{u_{k}(\bar{x})\right\}$. Therefore,

$$
\begin{aligned}
& \left\|\sum_{k=0}^{N}\left(\ln \left(\nu_{k+1}-\nu_{k}+1\right)\right)^{\sum_{j=1}^{m} \frac{1-\theta^{(2)}}{\theta_{j}^{(1)}}} \lambda_{\nu_{k}}^{\theta^{(2)}-1} \xi_{k}\right\|_{\bar{p}^{\prime}, \theta^{(2)^{\prime}}}^{*}<<
\end{aligned}
$$

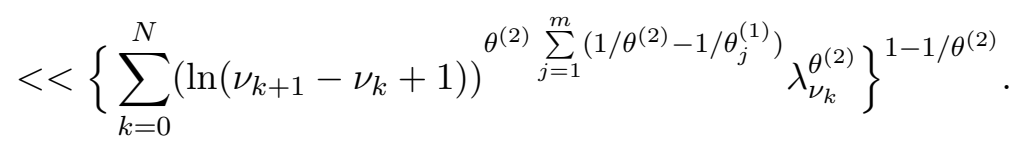

Now, it follows from inequalities (2.12), (2.13), and (2.14) that

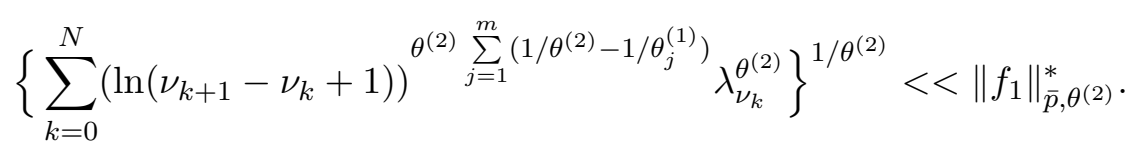

By (2.10), we find that $f_{1} \notin L_{\bar{p}, \theta^{(2)}}^{*}\left(\mathbb{I}^{m}\right), 1<\theta^{(2)}<\theta_{j}^{(1)}<+\infty, j=1, \ldots, m$. This contradicts the inclusion $E_{\bar{p}, \bar{\theta}^{(1)}}^{\lambda} \subset L_{\bar{p}, \theta^{(2)}}^{*}\left(\mathbb{I}^{m}\right)$. The proof of Theorem 3 is complete.

Remark 2. The results of L.A. Sherstneva [22] follow from Theorems 2 and 3 in the case $m=1$.

\section{Estimates of best approximations of functions with logarithmic smoothness}

Now, let us prove estimates of the value $E_{M}(F)_{\bar{p}, \bar{\theta}^{(2)}}$ for the classes $F=\mathbb{B}_{\bar{p}, \bar{\theta}^{(1)}}^{(0, \alpha)}$ and $F=\mathbb{A}_{\bar{p}, \bar{\theta}^{(1)}}^{(0, \gamma, \tau)}$.

Theorem 4. Let $1<p_{j}<\infty$ and $1 \leq \theta^{(2)}<\theta_{j}^{(1)}<\infty$ for $j=1, \ldots$, m, and let $1 \leq \tau \leq \infty$. If $\alpha>\sum_{j=1}^{m}\left(1 / \theta^{(2)}-1 / \theta_{j}^{(1)}\right)$, then $B_{\bar{p}, \overline{\theta^{(1)}}}^{(0, \alpha)} \subset L_{\bar{p}, \theta^{(2)}}^{*}\left(\mathbb{I}^{m}\right)$ and

$$
\|f\|_{\bar{p}, \theta^{(2)}}^{*}<<\|f\|_{B_{\bar{p}, \bar{\theta}}^{(0, \alpha, \tau)} .}
$$


P r o o f. Let $f \in B_{\bar{p}, \bar{\theta}^{(1)}}^{(0, \alpha)}$. Then, by the definition of the class, this function can be represented in the form of the series

$$
\sum_{\nu=0}^{\infty} Q_{2^{2^{\nu}}}(f, \bar{x}), \quad Q_{2^{2^{\nu}}}(f, \bar{x}) \in \mathfrak{F}_{\square_{2^{2}}}
$$

in the sense of convergence in the quasi-norm of the space $L_{\bar{p}, \bar{\theta}^{(1)}}^{*}\left(\mathbb{I}^{m}\right)$ and

$$
\left[\sum_{\nu=0}^{\infty}\left(2^{\nu \alpha}\left\|Q_{2^{2}}(f)\right\|_{\bar{p}, \bar{\theta}}^{*}\right)^{\tau}\right]^{1 / \tau}<+\infty
$$

If $\theta^{(2)}<\tau<\infty$, then, using the Hölder inequality and taking into account that $\alpha>\sum_{j=1}^{m}\left(1 / \theta^{(2)}-1 / \theta_{j}^{(1)}\right)$, we obtain

$$
\begin{gathered}
\left\{\sum_{\nu=0}^{\infty} 2^{\nu \sum_{j=1}^{m}\left(1 / \theta^{(2)}-1 / \theta_{j}^{(1)}\right) \theta^{(2)}}\left(\left\|Q_{2^{2}}(f)\right\|_{\bar{p}, \bar{\theta}^{(1)}}^{*}\right)^{\theta^{(2)}}\right\}^{1 / \theta^{(2)}} \leq \\
\leq\left\{\sum_{\nu=0}^{\infty} 2^{\nu \tau \alpha}\left(\left\|Q_{2^{2}}(f)\right\|_{\bar{p}, \bar{\theta}^{(1)}}^{*}\right)^{\tau}\right\}^{1 / \tau}\left\{\sum_{\nu=0}^{\infty} 2^{\nu \theta^{(2)} \beta^{\prime}\left(\sum_{j=1}^{m}\left(1 / \theta^{(2)}-1 / \theta_{j}^{(1)}\right)-\alpha\right)}\right\}^{\frac{1}{\theta^{(2)} \beta^{\prime}}} \leq \\
\leq C\left\{\sum_{\nu=0}^{\infty} 2^{\nu \tau \alpha}\left(\left\|Q_{2^{2}}(f)\right\|_{\bar{p}, \bar{\theta}^{(1)}}^{*}\right)^{\tau}\right\}^{1 / \tau},
\end{gathered}
$$

where

$$
\beta=\frac{\tau}{\theta^{(2)}}, \quad \beta^{\prime}=\frac{\beta}{\beta-1} .
$$

If $\tau=\infty$, then

$$
\begin{gathered}
\left\{\sum_{\nu=0}^{\infty} 2^{\nu \sum_{j=1}^{m}\left(1 / \theta^{(2)}-1 / \theta_{j}^{(1)}\right) \theta^{(2)}}\left(\left\|Q_{2^{2}}(f)\right\|_{\bar{p}, \bar{\theta}^{(1)}}^{*}\right)^{\theta^{(2)}}\right\}^{1 / \theta^{(2)}} \leq \\
\leq \sup _{\nu \in \mathbb{N}_{0}} 2^{\nu \alpha}\left\|Q_{2^{2}}(f)\right\|_{\bar{p}, \bar{\theta}^{(1)}}^{*}\left\{\sum_{\nu=0}^{\infty} 2^{\nu \theta^{(2)}\left(\sum_{j=1}^{m}\left(1 / \theta^{(2)}-1 / \theta_{j}^{(1)}\right)-\alpha\right)}\right\}^{1 / \theta^{(2)}} .
\end{gathered}
$$

If $\tau \leq \theta^{(2)}$, then, using the Jensen inequality (see [17, p. 125]), we obtain

$$
\left\{\sum_{\nu=0}^{\infty} 2^{\nu \sum_{j=1}^{m}\left(1 / \theta^{(2)}-1 / \theta_{j}^{(1)}\right) \theta^{(2)}}\left(\left\|Q_{2^{2}}(f)\right\|_{\bar{p}, \bar{\theta}^{(1)}}^{*}\right)^{\theta^{(2)}}\right\}^{1 / \theta^{(2)}} \leq\left\{\sum_{\nu=0}^{\infty} 2^{\nu \tau \alpha}\left(\left\|Q_{2^{2}}(f)\right\|_{\bar{p}, \bar{\theta}^{(1)}}^{*}\right)^{\tau}\right\}^{1 / \tau} .
$$

Thus, (3.1)-(3.3) imply that the series

$$
\sum_{\nu=0}^{\infty} 2^{\nu \sum_{j=1}^{m}\left(1 / \theta^{(2)}-1 / \theta_{j}^{(1)}\right) \theta^{(2)}}\left(\left\|Q_{2^{2^{\nu}}}(f)\right\|_{\bar{p}, \bar{\theta}^{(1)}}^{*}\right)^{\theta^{(2)}}
$$

is convergent for every function $f \in B_{\bar{p}, \bar{\theta}^{(1)}}^{(0, \alpha)}$.

Taking into account the monotonicity of the best approximation and the properties of the norm, it is easy to verify that 


$$
\begin{gathered}
\sum_{n=2}^{\infty} \frac{(\ln n)^{\sum_{j=1}^{m}\left(1 / \theta^{(2)}-1 / \theta_{j}^{(1)}\right)-1}}{n} E_{n, \ldots, n}^{\theta^{(2)}}(f)_{\bar{p}, \bar{\theta}^{(1)}}<<\sum_{\nu=0}^{\infty} 2^{\nu \sum_{j=1}^{m}\left(1 / \theta^{(2)}-1 / \theta_{j}^{(1)}\right) \theta^{(2)}} E_{2^{2^{\nu}}, \ldots, 2^{\nu}}^{\theta^{(2)}}(f)_{\bar{p}, \bar{\theta}^{(1)}}<< \\
<<\sum_{\nu=0}^{\infty} 2^{\nu \sum_{j=1}^{m}\left(1 / \theta^{(2)}-1 / \theta_{j}^{(1)}\right) \theta^{(2)}}\left(\left\|\sum_{l=\nu}^{\infty} Q_{2^{2^{l}}}(f)\right\|_{\bar{p}, \bar{\theta}^{(1)}}^{*}\right)^{\theta^{(2)}}<< \\
<<\sum_{\nu=0}^{\infty} 2^{\nu \sum_{j=1}^{m}\left(1 / \theta^{(2)}-1 / \theta_{j}^{(1)}\right) \theta^{(2)}}\left(\sum_{l=\nu}^{\infty}\left\|Q_{2^{2}}(f)\right\|_{\bar{p}, \bar{\theta}^{(1)}}^{*}\right)^{\theta^{(2)}} .
\end{gathered}
$$

Since $\theta^{(2)}<\theta_{j}^{(1)}, j=1, \ldots, m$, we have

$$
\sum_{\nu=0}^{n} 2^{\nu \sum_{j=1}^{m}\left(1 / \theta^{(2)}-1 / \theta_{j}^{(1)}\right) \theta^{(2)}}<<2^{n \sum_{j=1}^{m}\left(1 / \theta^{(2)}-1 / \theta_{j}^{(1)}\right) \theta^{(2)}}, \quad n \in \mathbb{N}_{0} .
$$

Therefore, according to [14, Lemma 2.2], we find from (3.5) that

$$
\sum_{n=2}^{\infty} \frac{(\ln n)^{\sum_{j=1}^{m}\left(1 / \theta^{(2)}-1 / \theta_{j}^{(1)}\right) \theta^{(2)}-1}}{n} E_{n, \ldots, n}^{\theta^{(2)}}(f)_{\bar{p}, \bar{\theta}^{(1)}}<<\sum_{\nu=0}^{\infty} 2^{\nu \sum_{j=1}^{m}\left(1 / \theta^{(2)}-1 / \theta_{j}^{(1)}\right) \theta^{(2)}}\left(\left\|Q_{2^{2}}(f)\right\|_{\bar{p}, \bar{\theta}^{(1)}}^{*}\right)^{\theta^{(2)}}
$$

Since the series (3.4) converges, it follows from (3.6) that

$$
\sum_{n=2}^{\infty} \frac{(\ln n)^{\sum_{j=1}^{m}\left(1 / \theta^{(2)}-1 / \theta_{j}^{(1)}\right) \theta^{(2)}-1}}{n} E_{n, \ldots, n}^{\theta^{(2)}}(f)_{\bar{p}, \bar{\theta}^{(1)}}<\infty .
$$

Hence, by Theorem 3 , we have $f \in L_{\bar{p}, \theta^{(2)}}^{*}\left(\mathbb{I}^{m}\right)$.

Let us estimate the quasi-norm $\|f\|_{\bar{p}, \bar{\theta}^{(1)}}^{*}$. By the quasi-norm property and the Hölder inequality, we obtain

$$
\begin{gathered}
\|f\|_{\bar{p}, \bar{\theta}^{(1)}}^{*}<<\sum_{\nu=0}^{\infty}\left\|Q_{2^{2}}(f)\right\|_{\bar{p}, \bar{\theta}^{(1)}}^{*}<< \\
<<\left(\sum_{\nu=0}^{\infty} 2^{\nu \sum_{j=1}^{m}\left(1 / \theta^{(2)}-1 / \theta_{j}^{(1)}\right) \theta^{(2)}}\left(\left\|Q_{2^{2^{\nu}}}(f)\right\|_{\bar{p}_{\bar{p}} \bar{\theta}^{(1)}}^{*}\right)^{\theta^{(2)}}\right)^{1 / \theta^{(2)}} .
\end{gathered}
$$

Therefore, according to relations (2.2), (3.6), and (3.7), we have

$$
\|f\|_{\bar{p}, \bar{\theta}^{(1)}}^{*}<<\left\{\sum_{\nu=0}^{\infty} 2^{\nu \sum_{j=1}^{m}\left(1 / \theta^{(2)}-1 / \theta_{j}^{(1)}\right) \theta^{(2)}}\left(\left\|Q_{2^{2}}(f)\right\|_{\bar{p}, \bar{\theta}^{(1)}}^{*}\right)^{\theta^{(2)}}\right\}^{1 / \theta^{(2)}} .
$$

Taking into account (3.1)-(3.3) and (3.8), we obtain

$$
\|f\|_{\bar{p}, \theta^{(2)}}^{*}<<\left\{\sum_{\nu=0}^{\infty} 2^{\nu \tau(\gamma+1 / \tau)}\left(\left\|Q_{2^{2}}(f)\right\|_{\bar{p}, \bar{\theta}^{(1)}}^{*}\right)^{\tau}\right\}^{1 / \tau}
$$

for every function $f \in B_{\bar{p}, \bar{\theta}^{(1)}}^{(0, \alpha)}$. The proof of Theorem 4 is complete. 
Theorem 5. Let $1<p_{j}<\infty$ and $1 \leq \theta^{(2)}<\theta_{j}^{(1)}<\infty$ for $j=1, \ldots$, m, and let $1 \leq \tau \leq \infty$. If $\alpha>\sum_{j=1}^{m}\left(1 / \theta^{(2)}-1 / \theta_{j}^{(1)}\right)$, then

$$
E_{M}\left(\mathbb{B}_{\bar{p}, \bar{\theta}^{(1)}}^{(0, \alpha)}\right)_{\bar{p}, \bar{\theta}^{(2)}} \asymp(\log (M+1))^{-\left(\alpha-\sum_{j=1}^{m}\left(1 / \theta^{(2)}-1 / \theta_{j}^{(1)}\right)\right)}, \quad M \in \mathbb{N} .
$$

P r o o f. Let $f \in \mathbb{B}_{\bar{p}, \bar{\theta}^{(1)}}^{(0, \alpha, \tau)}$. We have $\alpha>\sum_{j=1}^{m}\left(1 / \theta^{(2)}-1 / \theta_{j}^{(1)}\right) ;$ therefore, $f \in L_{\bar{p}, \theta^{(2)}}^{*}\left(\mathbb{I}^{m}\right)$ by Theorem 4 . Take a positive integer $l$ such that $2^{2^{l}} \leq M<2^{2^{l+1}}$. Then, using the best approximation property and inequality (3.9), we have

$$
E_{M}(f)_{\bar{p}, \theta^{(2)}} \leq E_{2^{2^{l}}}(f)_{\bar{p}, \theta^{(2)}}<<\left\{\sum_{\nu=l}^{\infty} 2^{\nu \sum_{j=1}^{m}\left(1 / \theta^{(2)}-1 / \theta_{j}^{(1)}\right) \theta^{(2)}}\left(\left\|Q_{2^{2^{\nu}}}(f)\right\|_{\bar{p}, \bar{\theta}^{(1)}}^{*}\right)^{\theta^{(2)}}\right\}^{1 / \theta^{(2)}} .
$$

If $\theta^{(2)}<\tau$, then by the Hölder inequality and in view of the fact that $\alpha>\sum_{j=1}^{m}\left(1 / \theta^{(2)}-1 / \theta_{j}^{(1)}\right)$, (3.10) implies that (see formula (3.1))

$$
\begin{gathered}
E_{M}(f)_{\bar{p}, \theta^{(2)}} \leq\left\{\sum_{\nu=0}^{\infty} 2^{\nu \tau \alpha}\left(\left\|Q_{2^{2}}(f)\right\|_{\bar{p}, \bar{\theta}^{(1)}}^{*}\right)^{\tau}\right\}^{1 / \tau} \times \\
\left\{\sum_{\nu=l}^{\infty} 2^{\nu \theta^{(2)} \beta^{\prime}\left(\sum_{j=1}^{m}\left(1 / \theta^{(2)}-1 / \theta_{j}^{(1)}\right)-\alpha\right)}\right\}^{\frac{1}{\theta^{(2)} \beta^{\prime}}}<<2^{-l\left(\alpha-\sum_{j=1}^{m}\left(1 / \theta^{(2)}-1 / \theta_{j}^{(1)}\right)\right)}
\end{gathered}
$$

for every function $f \in \mathbb{B}_{\bar{p}, \bar{\theta}^{(1)}}^{(0, \alpha,)}$ in the case $\theta^{(2)}<\tau$.

If $\tau \leq \theta^{(2)}$, then, arguing as in the proof of formula (3.3), by means of the Jensen inequality, we find from (3.10) that

$$
E_{M}(f)_{\bar{p}, \theta^{(2)}} \leq\left\{\sum_{\nu=0}^{\infty} 2^{\nu \tau \alpha}\left(\left\|Q_{2^{2^{\nu}}}(f)\right\|_{\bar{p}, \bar{\theta}^{(1)}}^{*}\right)^{\tau}\right\}^{1 / \tau} 2^{-l\left(\alpha-\sum_{j=1}^{m}\left(1 / \theta^{(2)}-1 / \theta_{j}^{(1)}\right)\right)}
$$

Now, taking into account that $2^{2^{l}} \leq M<2^{2^{l+1}}$, by formulas (3.11) and (3.12), we obtain

$$
E_{M}(f)_{\bar{p}, \theta^{(2)}}<<(\log (M+1))^{-\left(\alpha-\sum_{j=1}^{m}\left(1 / \theta^{(2)}-1 / \theta_{j}^{(1)}\right)\right)}
$$

for every function $f \in \mathbb{B}_{\bar{p}, \bar{\theta}^{(1)}}^{(0, \alpha)}$. Thus, the upper estimates are proved.

Let us prove the lower estimates. Consider the function

$$
f_{2}(\bar{x})=2^{-(n+1)\left(\alpha+\sum_{j=1}^{m} 1 / \theta_{j}^{(1)}\right)} \sum_{s=2^{n+1}+1}^{2^{n+2}} \sum_{\bar{k} \in \square_{2^{s}} \backslash \square_{2^{s-1}}} \prod_{j=1}^{m}\left(k_{j}-2^{s-1}+1\right)^{\frac{1}{p_{j}}-1} e^{i\langle\bar{k}, \bar{x}\rangle}
$$

where $\bar{x} \in \mathbb{I}^{m}$ and $n \in \mathbb{N}_{0}$. It is well known that

$$
\left\|\sum_{s=2^{n+1}}^{2^{n+2}} \sigma_{s}\left(f_{2}\right)\right\|_{\bar{p}, \bar{\theta}^{(1)}}^{*}=2^{-(n+1)\left(\alpha+\sum_{j=1}^{m} 1 / \theta_{j}^{(1)}\right)}\left\|\sum_{s=2^{n+1}+1}^{2^{n+2}} \sum_{\bar{k} \in \square_{2} s \backslash \square_{2^{s-1}}} \prod_{j=1}^{m}\left(k_{j}-2^{s-1}+1\right)^{\frac{1}{p_{j}}-1} e^{i\langle\bar{k}, \bar{x}\rangle}\right\|_{\bar{p}, \bar{\theta}^{(1)}}^{*}<<
$$




$$
<<2^{-(n+1)\left(\alpha+\sum_{j=1}^{m} 1 / \theta_{j}^{(1)}\right)}\left(\log \left(2^{2^{n+2}}-2^{2^{n+1}}\right)\right)^{\sum_{j=1}^{m} 1 / \theta_{j}^{(1)}}<<2^{-(n+1) \alpha} .
$$

Thus,

$$
\left\{\sum_{\nu=0}^{\infty} 2^{\nu \tau \alpha}\left(\left\|\sum_{s=2^{\nu}}^{2^{\nu+1}} \sigma_{s}\left(f_{2}\right)\right\|_{\bar{p}, \bar{\theta}^{(1)}}^{*}\right)^{\tau}\right\}^{1 / \tau}=2^{(n+1) \alpha}\left\|\sum_{s=2^{n+1}}^{2^{n+2}} \sigma_{s}\left(f_{2}\right)\right\|_{\bar{p}, \bar{\theta}^{(1)}}^{*} \leq C_{1} .
$$

Hence, $C_{1}^{-1} f_{2} \in \mathbb{B}_{\bar{p}, \bar{\theta}^{(1)}}^{(0, \alpha)}$ for $1<\theta^{(2)}<\infty$ and $1 \leq \tau<\infty$. Next, by the definition of the best approximation and the estimate

$$
\left\|\sum_{s=2^{n+1}+1}^{2^{n+2}} \sum_{\bar{k} \in \square_{2} \backslash \square_{2^{s-1}}} \prod_{j=1}^{m}\left(k_{j}-2^{s-1}+1\right)^{\frac{1}{p_{j}}-1} e^{i\langle\bar{k}, \bar{x}\rangle}\right\|_{\bar{p}, \theta^{(2)}}^{*}>>2^{n \frac{m}{\theta^{(2)}}},
$$

we have

$$
\begin{aligned}
& E_{2^{2^{n}}}\left(f_{2}\right)_{\bar{p}, \theta^{(2)}}=C_{1}^{-1}\left\|f_{2}\right\|_{\bar{p}, \theta^{(2)}}^{*}= \\
& =C_{1}^{-1} 2^{-(n+1)\left(\alpha+\sum_{j=1}^{m} 1 / \theta_{j}^{(1)}\right)}\left\|\sum_{s=2^{n+1}+1}^{2^{n+2}} \sum_{\bar{k} \in \square_{2^{s}} \backslash \square_{2^{s-1}}} \prod_{j=1}^{m}\left(k_{j}-2^{s-1}+1\right)^{\frac{1}{p_{j}}-1} e^{i\langle\bar{k}, \bar{x}\rangle}\right\|_{\bar{p}, \theta^{(2)}}^{*}>> \\
& >>2^{-(n+1)\left(\alpha-\sum_{j=1}^{m}\left(1 / \theta^{(2)}-1 / \theta_{j}^{(1)}\right)\right)} .
\end{aligned}
$$

Taking into account that $2^{2^{n}} \leq M<2^{2^{n+1}}$, we obtain

$$
E_{M}\left(f_{2}\right)_{\bar{p}, \theta^{(2)}}>>(\log (M+1))^{\left(\alpha-\sum_{j=1}^{m}\left(1 / \theta^{(2)}-1 / \theta_{j}^{(1)}\right)\right)}
$$

for $1 \leq \theta^{(2)}<\infty$ and $1 \leq \tau \leq \infty$. Thus, the proof of Theorem 5 is compete.

Theorem 6. Let $1<p_{j}<\infty$ and $1 \leq \theta^{(2)}<\theta_{j}^{(1)}<\infty$ for $j=1, \ldots, m$, and let $1 \leq \tau \leq \infty$. If $\gamma>\sum_{j=1}^{m}\left(1 / \theta^{(2)}-1 / \theta_{j}^{(1)}\right)-1 / \tau$, then

$$
E_{M}\left(\mathbb{A}_{\bar{p}, \bar{\theta}^{(1)}}^{(0, \gamma, \tau)}\right)_{\bar{p}, \bar{\theta}^{(2)}} \asymp(\log (M+1))^{-\left(\gamma+1 / \tau-\sum_{j=1}^{m}\left(1 / \theta^{(2)}-1 / \theta_{j}^{(1)}\right)\right)} .
$$

P r o o f. Since $\mathbb{A}_{\bar{p}, \bar{\theta}^{(1)}}^{(0, \gamma)}$ and $\mathbb{B}_{\bar{p},,^{(1)}}^{(0, \gamma+1 / \tau, \tau)}$ coincide, the statement of Theorem 6 follows from Theorem 5 .

\section{Conclusion}

The best approximations of functions of the classes $\mathbb{B}_{\bar{p}, \bar{\theta}^{(1)}}^{(0, \alpha)}$ and $\mathbb{A}_{\bar{p}, \bar{\theta}^{(1)}}^{(0, \gamma, \tau)}$ in the space $L_{\bar{p}, \theta^{(2)}}^{*}\left(\mathbb{I}^{m}\right)$ have logarithmic order.

Note that estimates of the quantities $E_{M}\left(\mathbb{B}_{\bar{p}, \bar{\theta}^{(1)}}^{(0, \gamma, \tau)}\right)_{\bar{p}, \bar{\theta}^{(2)}}$ and $E_{M}\left(\mathbb{A}_{\bar{p}, \bar{\theta}^{(1)}}^{(0, \gamma, \tau)}\right)_{\bar{p}, \bar{\theta}^{(2)}}$ are unknown in the case $\theta_{j}^{(1)}=\theta^{(2)}, j=1, \ldots, m$. 


\section{REFERENCES}

1. Akishev G. A. On imbedding of some classes of functions of several variables into the Lorentz space. Izv. Akad. Nauk Kaz. SSR, Ser. Fiz.-Mat., 1982. No. 3. P. 47-51. (in Russian)

2. Akishev G. The estimates of approximations classes in the Lorentz space. AIP Conf. Proc., 2015. Vol. 1676, No. 1. Art. no. 020027. P. 1-4. DOI: 10.1063/1.4930453

3. Akishev G. An inequality of different metric for multivariate generalized polynomials. East J. Approx., 2006. Vol. 12, No. 1. P. 25-36.

4. Akishev G. A. Estimates for best approximations of functions from the logarithmic smoothness class in the Lorentz space. Trudy Inst. Mat. Mekh. UrO RAN, 2017. Vol. 23, No. 3. P. 3-21. DOI: 10.21538/0134-4889-2017-23-3-3-21 (in Russian)

5. Andrienko V. A. The imbedding of certain classes of functions. Math. USSR-Izv., 1967. Vol. 1, No. 6. P. $1255-1270$.

6. Bekmaganbetov K. A. About order of approximation of Besov classes in metric of anisotropic Lorentz spaces. Ufimsk. Mat. Zh., 2009. Vol. 1, No. 2. P. 9-16. (in Russian)

7. Bekmaganbetov K. A. Order of approximation of Besov classes in the metric of anisotropic Lorentz spaces. In: Methods of Fourier Analysis and Approximation Theory. Ruzhansky M., Tikhonov S. (eds.) Appl. Numer. Harmon. Anal. Cham: Birkhäuser, 2016. P. 149-158. DOI: 10.1007/978-3-319-27466-9_10

8. Blozinski A.P. Multivariate rearrangements and Banach function spaces with mixed norms. Trans. Amer. Math. Soc., 1981. Vol. 263, No. 1. P. 149-167. DOI: 10.2307/1998649

9. Cobos F., Domínguez Ó. On Besov spaces of logarithmic smoothness and Lipschitz spaces. J. Math. Anal. Appl., 2015. Vol. 425, No. 1. P. 71-84. DOI: 10.1016/j.jmaa.2014.12.034

10. Cobos F., Domínguez Ó. Approximation spaces, limiting interpolation and Besov spaces. J. Approx. Theory, 2015. Vol. 189. P. 43-66. DOI: 10.1016/j.jat.2014.09.002

11. DeVore R.A., Lorentz G.G. Constructive Approximation. Grundlehren Math. Wiss., vol. 33. Berlin, Heidelberg: Springer-Verlag, 1993. 453 p.

12. Dinh Dũng, Temlyakov V.N., Ullrich T. Hyperbolic Cross Approximation. 2016. 154 p. arXiv:1601.03978v1 [math.NA]

13. Domínguez Ó., Tikhonov S. Function Spaces of Logarithmic Smoothness: Embeddings and Characterizations. 2018. 162 p. arXiv:1811.06399v2 [math.FA]

14. Johansson H. Embedding of $H_{p}^{\omega}$ in Some Lorentz Spaces. Research Reports. Univ. Umeå, 1975. Report No. 6.26 p.

15. Kolyada V. I. Imbedding theorems and inequalities in various metrics for best approximations. Mat. Sb. (N.S.), 1977. Vol. 102 (144), No. 2. P. 195-215. (in Russian)

16. Kolyada V.I. Rearrangements of functions and embedding theorems. Russian Math. Surveys, 1989. Vol. 44, No. 5 P. 73-117. DOI: 10.1070/RM1989v044n05ABEH002287

17. Nikol'skii S. M. Priblizhenie funkcij mnogih peremennyh i teoremy vlozheniya [Approximation of Function of Several Variables and Imbedding Theorems]. Moscow: Nauka, 1977. 456 p. (in Russian)

18. Nursultanov E. D. Interpolation theorems for anisotropic function spaces and their applications. Dokl. Akad. Nauk RAN, 2004. Vol. 394, No. 1. P. 22-25.

19. Nursultanov E. D. Nikol'skii's inequality for different metrics and properties of the sequence of norms of the Fourier sums of a function in the Lorentz space. Proc. Steklov Inst. Math., 2006. Vol. 255. P. 185-202. DOI: $10.1134 /$ S0081543806040158

20. Pietsch A., Approximation spaces. J. Approx. Theory, 1981. Vol. 32, No. 2. P. 115-134. DOI: 10.1016/0021-9045(81)90109-X

21. Romanyuk A. S. Approximation of the isotropic classes $B_{p, \theta}^{r}$ of periodic functions of several variables in the space $L_{q}$. Zb. Pr. Inst. Mat. NAN Ukr., 2008. Vol. 5, No. 1. P. 263-278. (in Russian)

22. Sherstneva L. A. On the properties of best Lorentz approximations, and certain embedding theorems. Soviet Math. (Iz. VUZ), 1987. Vol. 31, No. 10. P. 62-73.

23. Smailov E. S., Akishev G. Embedding theorems in the Lorentz space and their applications. Izv. Akad. Nauk Kaz. SSR, Ser. Fiz.-Mat., 1984. No. 1. P. 66-70. (in Russian)

24. Stasyuk S. A. Approximating characteristics of the analogs of Besov classes with logarithmic smoothness. Ukr. Math. J., 2014. Vol. 66, No. 4, P. 553-560. DOI: 10.1007/s11253-014-0952-5 
25. Stasyuk S. A. Kolmogorov widths for analogs of the Nikol'skii-Besov classes with logarithmic smoothness. Ukr. Math. J., 2015. Vol. 67, No. 11. P. 1786-1792. DOI: 10.1007/s11253-016-1190-9

26. Storozhenko È. A. Embedding theorems and best approximations. Math. USSR-Sb., 1975. Vol. 26 , No. 2. P. 213-224. DOI: 10.1070/SM1975v026n02ABEH002477

27. Temirgaliev N. Embedding in some Lorentz spaces. Izv. Vyssh. Uchebn. Zaved. Mat., 1980. No. 6. P. 8385. (in Russian)

28. Temirgaliev N. Embeddings of the classes $H_{p}^{\omega}$ in Lorentz spaces. Sib. Math. J., 1983. Vol. 24, No. 2. P. 287-298. DOI: 10.1007/BF00968743

29. Temlyakov V. Multivariate Approximation. Cambridge University Press. $2018 . \quad 551 \quad$ p. DOI: $10.1017 / 9781108689687$

30. Ul'janov P.L. The imbedding of certain function classes $H_{p}^{\omega}$. Math. USSR-Izv., 1968. Vol. 2 , No. 3. P. 601-637. DOI: 10.1070/IM1968v002n03ABEH000650 